\title{
TIME SERIES MODELLING OF THE KOBE-OSAKA EARTHQUAKE RECORDINGS
}

\author{
N. SINGH, D. TAMPUBOLON, and V. S. S. YADAVALLI
}

Received 30 April 2001

\begin{abstract}
A problem of great interest in monitoring a nuclear test ban treaty (NTBT) is related to interpreting properly the differences between a waveform generated by a nuclear explosion and that generated by an earthquake. With a view of comparing these two types of waveforms, Singh (1992) developed a technique for identifying a model in time domain. Fortunately this technique has been found useful in modelling the recordings of the killer earthquake occurred in the Kobe-Osaka region of Japan at 5.46 am on 17 January, 1995. The aim of the present study is to show how well the method for identifying a model (developed by Singh (1992)) can be used for describing the vibrations of the above mentioned earthquake recorded at Charters Towers in Queensland, Australia.
\end{abstract}

2000 Mathematics Subject Classification: 62P05, 62M10.

1. Introduction. Many researchers have studied the structure of seismic records. Most of these studies (with the exception of Tjostheim [3, 4] and Dargahi-Noubery et al. [2]) have been carried out in the frequency domain and they have been mainly concerned with the properties of their spectra.

The purpose of the present study is to model the recordings of the earthquake recently occurred at the Kobe-Osaka region of Japan on 17 January, 1995, in time domain, that is, to fit an autoregressive moving average model to the seismogram (see Figure 1.1) recorded at Charters Towers, Queensland, Australia.

One of the advantages of fitting a parametric model is that if a good model can be fitted, the process can be characterized by numerical values of a few parameters as opposed to the conjectural interpretation of the plots of spectra. Other advantages of the parametric modelling a time series in general have been highlighted by Box and Jenkins [1].

It has been found (see Tjostheim [3, 4], Dargahi-Noubary et al. [2]) that the lowerorder autoregressive (AR) models are often appropriate for short period seismograms. Let $Y(t)$ be a wide-sense-stationary (wss) process in discrete time. The process is said to be an AR process of order $p$ (abbreviated $\operatorname{AR}(p)$ ) if $Y(t)$ satisfies the difference equation

$$
\varphi(B) Y(t)=\varepsilon(t)
$$

where $\varphi(B)=1-\varphi_{1} B-\varphi_{2} B^{2}-\cdots-\varphi_{p} B^{p}$ is a polynomial in $B$ of degree $p$ and is called the AR operator of order $p$, the $\varphi^{\prime}$ 's are constant coefficients; $B$ is the backward shift operator defined by $B^{j} Y(t)=Y(t-j)$; $\varepsilon(t)$ is a wss process such that $E\{\varepsilon(t) \varepsilon(s)\}=\sigma_{\varepsilon}^{2} \delta_{t s}$, where $\delta_{t s}$ is the Kronecker delta. Furthermore, it is assumed 


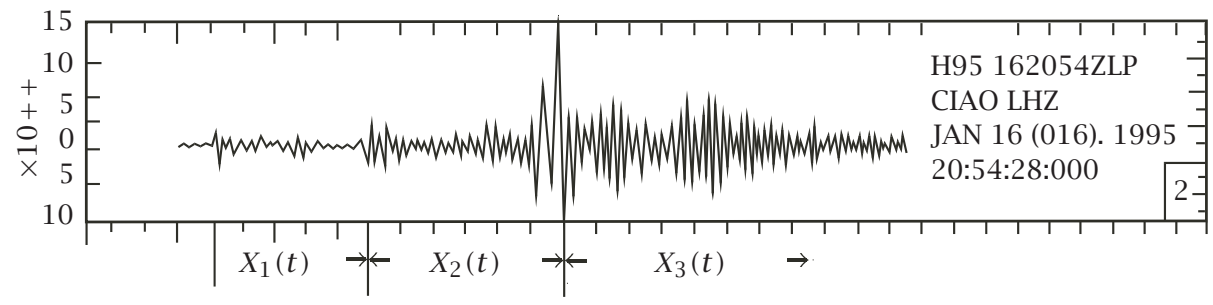

FIGURE 1.1. Seismogram of the Kobe earthquake of 17 January, 1995 recorded at Charters Towers.

that $E\{Y(t) \varepsilon(s)\}=0$, for $s>t$. Obviously, $Y(t)$ has zero mean (an assumption valid for seismograms). Notice that model (1.1) contains $p+1$ parameters, namely, the $\varphi_{i}$ 's, $i=1, \ldots, p$ and $\sigma_{\varepsilon}^{2}$. Once the order $p$ is determined, the standard methods for estimating parameters can be used.

It is well known that a seismogram recorded over its entire range is zero-mean stationary in some sections while it is nonstationary-in-variance in others.

It may be noticed that the pattern of the series in Figure 1.1 is of this type. We divide the whole seismogram into three subseries and denote them by $X_{1}(t), X_{2}(t)$, and $X_{3}(t)$ as shown in Figure 1.1. Thus we have three series out of the seismogram in Figure 1.1. Then a model of the following type may be fitted to the seismogram, namely

$$
Z(t)=\sum_{j=1}^{3} \delta_{i j} X_{i}(t), \quad \delta_{i j}= \begin{cases}1, & \text { if } i=j \\ 0, & \text { otherwise }\end{cases}
$$

(see Figure 1.1). Series $X_{1}(t), X_{2}(t)$, and $X_{3}(t)$ consist of 670,535 , and 1051 observations, respectively, and each is assumed to follow an ARMA $(p, 0)$ process.

Now, the problem is to identify the model for each of these series and then to estimate the parameters.

Before discussing these problems in Sections 3 and 4, we review briefly the related work done previously in time domain, for information and ready reference to the reader.

2. Short review of previous studies of seismograms in time domain. Tjostheim $[3,4]$ considered the AR model defined by

$$
X(t)=\sum_{j=1}^{p} \alpha_{j}(t) X(t-j)=U(t),
$$

where $X(t)$ denotes the seismic noise recorded at NORSAR, $E(U(t))=0$, $\operatorname{Var}(U(t))=$ $\sigma_{u}^{2}(t)$. It may be noted in model (2.1) that the coefficients $\alpha_{j}(t), j=1, \ldots, p$, and the residual variance $\sigma_{u}^{2}(t)$ are assumed to be functions of $t$, though without any specific forms. They were estimated using two different samples and shown to be significantly different from the two samples indicating their time dependence. 
On the other hand, Dargahi-Noubary et al. [2] considered a model of the type

$$
X(t)=\sum_{j=1}^{p} \alpha_{j}(k) X(t-j)=U(t),
$$

where $\alpha_{j}(k)=(-1)^{j}\left(\begin{array}{c}p \\ j\end{array}\right) \exp (-j k), j=1,2, \ldots, p$, and $U(t) \sim N\left(0, \sigma_{u}^{2}\right)$.

Thus model (2.2) consists of only two parameters, that is, $k$ and $\sigma_{u}^{2}$. The maximum likelihood estimators of these parameters have been obtained.

However, in neither of these studies, nothing seems to have taken into account the fact that the series has increasing (or decreasing) variance in a certain range of the series.

It is this problem which has been addressed in this paper. A realistic approach is suggested in the following sections with an application to the Kobe-Osaka earthquake recordings.

3. AR models with time-dependent error variance. Let $(\Omega, \beta, p)$ denote a probability space and let $\mathscr{L}(\cdot)=\mathscr{L}(\Omega, \beta, p)$ denote the space of all real-valued random variables on $(\Omega, \beta, p)$ with zero mean and finite second-order moments. The space $\mathscr{L}(\cdot)$ is called the Hilbert space if the inner product and norm are defined, respectively, by

$$
\langle\xi, \eta\rangle=E(\xi, \eta), \quad\|\xi\|^{2}=E\left(\xi^{2}\right), \quad \xi, \eta \in \mathscr{L}(\cdot) .
$$

Consider now a zero-mean $\operatorname{AR}(P)$ model with time-dependent error variance defined by

$$
\varphi(B) X(t)=w(t),
$$

where $\alpha(B)$ is the AR operator and $w(t) \in \mathscr{L}(\cdot)$ is a white-noise with zero mean and time-dependent variance $E\left\{w^{2}(t)\right\}=\sigma_{w}^{2}(t)$ such that $0<k \leq E\left\{w^{2}(t)\right\} \leq K$, where $k$ and $K$ are finite positive constants. Model (2.1) is stationary if, (i) all roots of equation $\alpha(x)=0$ lie outside the unit circle and (ii) the noise process $w(t)$ is stationary, that is, $E\left\{w^{2}(t)\right\}=\sigma_{w}^{2}$, a constant. It follows that $E(X(t))=0$ and $E X^{2}(t)<\infty$.

Put $\sigma_{w}^{2}(t)=H_{w}(t)$, where $H_{w}(t)$ is a positive function defined over the interval $\left[t_{1} \leq t \leq t_{2}\right]$. It is assumed that $H_{w}(t)$ is bounded and bounded away from zero, that is, $0<k \leq H_{w}(t) \leq K$, where $k<K$ are positive constants;

$$
E[X(t) w(s)]=0 \text { for } t<s, t, s \in\left[t_{1}, t_{2}\right] .
$$

Furthermore, we are interested in the family for which $H_{w}(t)$ is a parametric function of $t$. For all positive integer values of $t$, if the same parametric function is used, the class of possible functions will be restricted to the family such that $H_{w}(t) \geq 0$.

REMARK 3.1. It is interesting to note the relationship between $\operatorname{Var} X_{t}=H_{X}(t)$ and $\operatorname{Var} W_{t}=H_{w}(t)$ in the interval $\left[t_{1}, t_{2}\right]$ as shown below: for convenience let $t_{1}=0$, $t_{2}=t$, then for an $\operatorname{AR}(1)$, it can be seen that

$$
H_{X}(t)=\varphi^{2 t} H_{X}(0)+H_{w}(t)+\sum_{j=1}^{2 t-1} \varphi^{2 j-1} H_{w}(t-j) .
$$


Notice that for $|\phi|<1$, and $t \rightarrow \infty, H_{X}(t)$ will depend on the last two terms in (3.2) and hence the form of $H_{X}(t)$ will be similar to that of $H_{w}(t)$. For instance if $H_{w}(t)$ is a linear function of $t, H_{X}(t)$ will also be a linear function of $t$, approximately.

Let $X^{*}(t)$ be a uniquely determined bounded solution of (3.2) and let $\mu\left(X^{*}(t)\right)$ (resp., $\mu(w(t))$ ) denote a closed linear subspace in $\mathscr{L}(\cdot)$ spanned by all the random variables $X^{*}(t)$ (resp., $w(t)$ ). Similarly, let $\mu_{\tau}^{*}\left(X^{*}(t)\right)$ (resp., $\mu_{\tau}\left(X^{*}(t)\right)$ ) denote the closed linear subspace in $\mathscr{L}(\cdot)$ spanned by all variables $X^{*}(t), t \leq \tau$ (resp., $w(t)$, $t \leq \tau$ ). Suppose that $Y^{*}(t)$ is a uniquely determined stationary solution to (1.1), then there exists a uniquely determined bounded operator $S(t)$ such that

$$
S(t): \mu\left(Y^{*}(t)\right) \longrightarrow \mu\left(X^{*}(t)\right)
$$

with a bounded inverse $S^{-1}(t): \mu\left(X^{*}(t)\right) \rightarrow \mu\left(Y^{*}(t)\right)$ so that

$$
X^{*}(t)=S(t) Y^{*}(t) \quad \forall t .
$$

THEOREM 3.2. Let $X^{*}(t)$ and $Y^{*}(t)$ be defined as above. Then it follows from (3.6) that

(i) $E\left(X^{*}(t)\right)=S(t) E Y^{*}(t)$;

(ii) $\operatorname{Var}\left(X^{*}(t)\right)=S^{2}(t) \operatorname{Var} Y^{*}(t)$;

(iii) $\rho_{X}(t, s)=\rho_{Y}|t-s|$.

The proof is simple and straightforward.

REMARK 3.3. It may be noticed from Theorem 3.2(iii) that the correlation structure of $X(t)$ is the same as that of the zero-mean stationary process $\{Y(t)\}$; although $X(t)$ is zero-mean nonstationary-in-variance.

It is this common characteristic of the two processes which has been exploited in the sequel for the identification of both $X(t)$ and $Y(t)$ as well as the identification of the covariance structure of the white-noise $w(t)$ in (3.2).

For example, let $\{Y(t)\}$ be a zero-mean stationary $\operatorname{AR}(2)$ process defined by

$$
Y(t)=\varphi_{1} Y(t-1)+\varphi_{2} Y(t-2)+\varepsilon(t) ; \quad \varepsilon(t) \sim N\left(0, \sigma_{\varepsilon}^{2}\right) .
$$

Under the transformation $X(t)=S(t) Y(t)$, we have

$$
X(t)=\varphi_{1}(t) X(t-1)+\varphi_{2}(t) X(t-2)+w(t),
$$

where

$$
\varphi_{1}(t)=\frac{\varphi_{1} S(t)}{S(t-1)}, \quad \varphi_{2}=\frac{\varphi_{2} S(t)}{S(t-1)}, \quad w(t)=S(t) \varepsilon(t) .
$$

In general, it can be seen that

$$
\lim _{t \rightarrow \infty} \varphi_{i}(t) \longrightarrow \varphi_{i}, \quad i=1,2 .
$$

In particular, let $S(t)=\exp (\alpha t)$, then we have

$$
X(t)=\varphi_{1}^{*} X(t-1)+\varphi_{2}^{*} X(t-2)+w(t),
$$


where

$$
\begin{gathered}
\varphi_{1}^{*}=\varphi_{1} e^{\alpha}, \quad \varphi_{2}^{*}=\varphi_{e} e^{2 \alpha}, \\
H_{w}(t)=\operatorname{Var}(w(t))=\sigma_{\varepsilon}^{2} e^{2 \alpha-t}=\beta e^{c-t}, \text { say },
\end{gathered}
$$

where

$$
\beta=\sigma_{\varepsilon}^{2}, \quad c=2 \alpha
$$

EXAMPLE 3.4. Take $\varphi_{1}=-0.55, \varphi_{2}=0.35, \beta=1, \alpha=0.02$, then

$$
\begin{aligned}
Y(t) & =-0.55 Y(t-1)+0.35 Y(t-2)+\varepsilon(t), \\
S(t) & =\exp (0.02 t) \\
X(t) & =\exp (0.02 t) Y(t)
\end{aligned}
$$

and hence

$$
X(t)=-0.56 X(t-1)+0.36 X(t-2)+w(t)
$$

where

$$
w(t)=\exp (0.02 t) \varepsilon(t), \quad H_{w}(t)=\exp (0.04 t) \sigma_{\varepsilon}^{2} .
$$

A sample $\{Y(1), \ldots, Y(200)\}$ of 200 values was generated using (3.14) on VAX C and then a sample $\{X(1), \ldots, X(200)\}$ was generated using (3.16). These samples along with their respective ACF's and PACF's are plotted in Figures 3.1a and 3.1b.

3.1. Inference and conclusions. It may be noticed from Figure 3.1 that

(i) the series $Y(t)$ is stationary as expected;

(ii) the series $X(t)$ under the transformation (3.16) is a naturally exponentially increasing series since the variance of the white-noise $w(t)$ is assumed to be an exponential function of $t$;

(iii) the ACF's and PACF's of both $Y(t)$ and $X(t)$ are the same as expected (see Theorem 3.2), except for some sampling fluctuations;

(iv) both ACF and PACF of $X(t)$ (and/or $Y(t)$ ) suggest that the underlying stationary process $Y(t)$ is an $\mathrm{AR}(2)$ and the oscillatory nature of the ACF of either $X(t)$ or $Y(t)$, that one of the coefficients of the process $Y(t)$, is negative.

4. Preliminary identification. Given a zero-mean and nonstationary-in-variance series such as $X(t)$ in the preceding section, the following steps are suggested to identify the process:

(1) plot the series, its ACF and PACF,

(2) if the plot of the series is exponentially increasing (or decreasing) on both sides of the mean then it may be argued that the error-variance is an exponential function of time $t$ with positive (or negative) exponent. Similar interpretation may be given to the series if it is linearly increasing (or decreasing),

(3) the ACF and PACF together would suggest the order of the underlying stationary process such as the $Y(t)$ in the above example, 

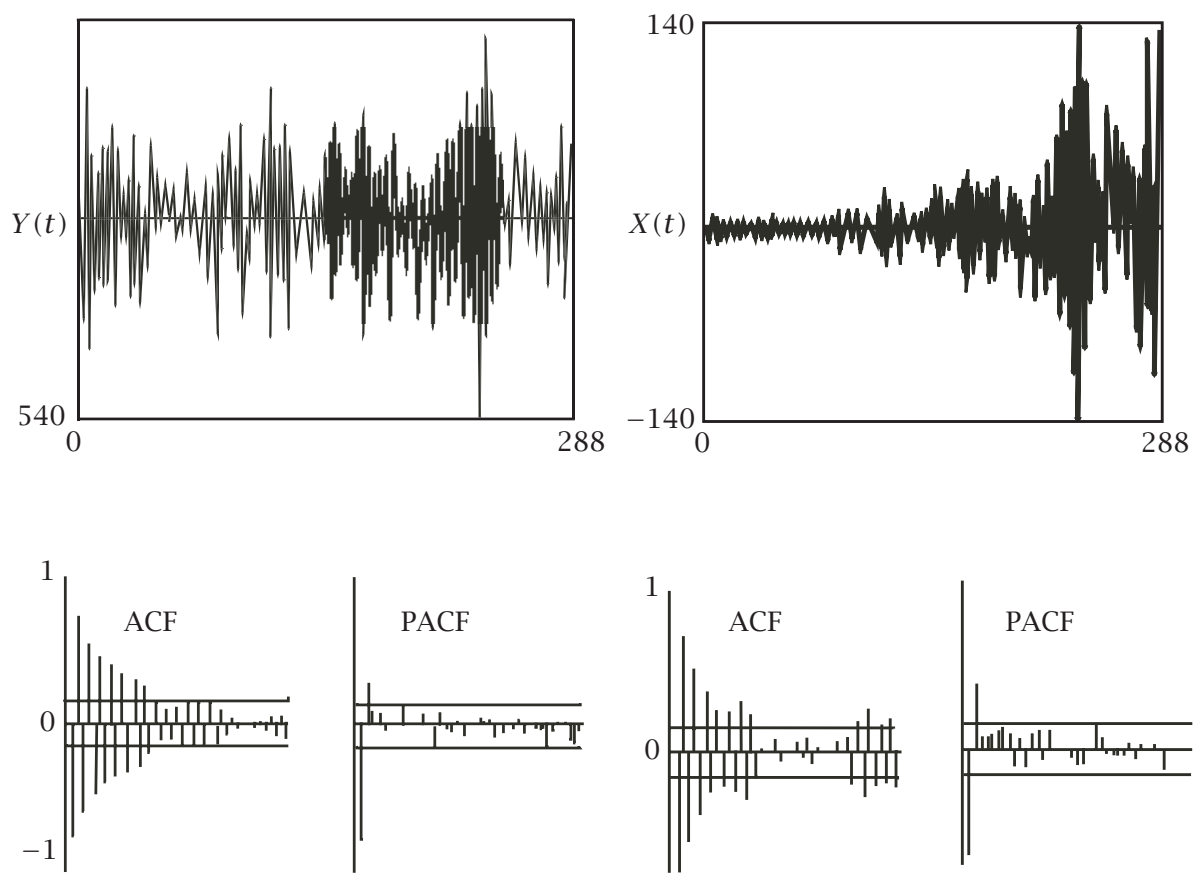

(a) Plots of $Y(t)$, its ACF and PACF.

(b) Plots of $X(t)$, its ACF and PACF.

FIGURE 3.1

(4) thus having identified the order of the AR process that can be fitted to the given $X(t)$ series and the covariance structure of the white-noise, any standard procedure of estimation such as the maximum likelihood (ML) can be used to estimate the parameters in the model and the covariance function of the whitenoise.

4.1. Advantages. One of the advantages of the above procedure is that it enables us to estimate the variance of the white-noise and the coefficients of the underlying stationary process which is referred to as $Y(t)$ in the preceding example. For instance, let $Y(t)$ be the underlying stationary $\operatorname{AR}(1)$ defined by

$$
Y(t)=\varphi Y(t-1)+\varepsilon_{t}, \quad \varepsilon_{t} \sim N\left(0, \sigma_{\varepsilon}^{2}\right),
$$

and let

$$
X(t)=S(t) Y(t),
$$

then

$$
X(t)=\varphi(t) X(t-1)+w(t),
$$


where

$$
\varphi(t)=\frac{\varphi S(t)}{S(t-1)}, \quad w(t)=S(t) \varepsilon(t) .
$$

Assuming $S(t)=\exp (\alpha t)$, we have $\varphi(t)=\varphi e^{\alpha}=\varphi^{*}$

$$
H_{w}(t)=\operatorname{Var} w(t)=\exp (2 \alpha t) \sigma_{\varepsilon}^{2}=\beta \exp (c t),
$$

where $\beta=\sigma_{\varepsilon}^{2}$ and $c=2 \alpha$. Given $X(1), \ldots, X(n)$, the ML method will yield the estimators of $\varphi^{*}, \beta$ and thus enabling us to estimate $\sigma_{\varepsilon}^{2}$, the coefficients of models $Y(t)$ and $X(t)$ in (4.1) and (4.3), respectively.

5. Fitting models to the Kobe-Osaka seismic recordings. The seismogram of the Kobe earthquake recorded at Charters Towers is given in Figure 1.1. The graph displays the vertical component of the ground displacement (in $\mathrm{nm}$ ) versus time in minutes. The total number of observations recorded were 2256. This entire set of observations has been divided into three main parts. We denote them by $X_{1}(t), X_{2}(t)$, and $X_{3}(t)$ series as shown in Figure 1.1. These series consist of 670, 535, and 1051 observations, respectively.

5.1. Modelling of series $X_{2}(t)$. First of all, we study and model series $X_{2}(t)$ due to its unusual appearance. This consists of 535 values and it is displayed in Figure 5.1.

The ACF and PACF of $X_{2}(t)$ are shown in Figure 5.2.

INFERENCE. (i) From Figure 5.3, we infer that the variance structure of the series $X_{2}(t)$ is time-dependent and seems to be an exponential function of $t$ multiplied by a constant which may be taken as an error-variance of the underlying process $Y_{2}(t)$. (See Section 4.)

(ii) Both ACF and PACF suggest that the representative process is pure autoregressive process of order three $(R(3))$.

Based on this preliminary inference, we postulate the underlying stationary process of the type

$$
Y_{2}(t)=\varphi_{1} Y_{2}(t-1)+\varphi_{2} Y_{2}(t-2)+\varphi_{3} Y_{2}(t-3)+\varepsilon_{3}(t)
$$

where $\varepsilon_{3}(t) \sim N\left(0, \sigma_{\varepsilon}^{2}\right)$, and select $S(t)=\exp (\alpha t)$.

Put

$$
X_{2}(t)=\exp (\alpha t) Y_{2}(t)
$$

Then the model for $X_{2}(t)$ may be defined by

$$
X_{2}(t)=\varphi_{1}(t) X_{2}(t-1)+\varphi_{2}(t) X_{2}(t-2)+\varphi_{3}(t) X_{3}(t-3)+w(t),
$$

where

$$
\begin{aligned}
\varphi_{1}(t)=\varphi_{1}^{*}=\varphi_{1} e^{\alpha}, \quad \varphi_{2}(t) & =\varphi_{2}^{*}=\varphi_{2} e^{2 \alpha}, \quad \varphi_{3}(t)=\varphi_{3}^{*}=\varphi_{3} e^{3 \alpha}, \\
w(t) & =\exp (\alpha t) \varepsilon(t),
\end{aligned}
$$




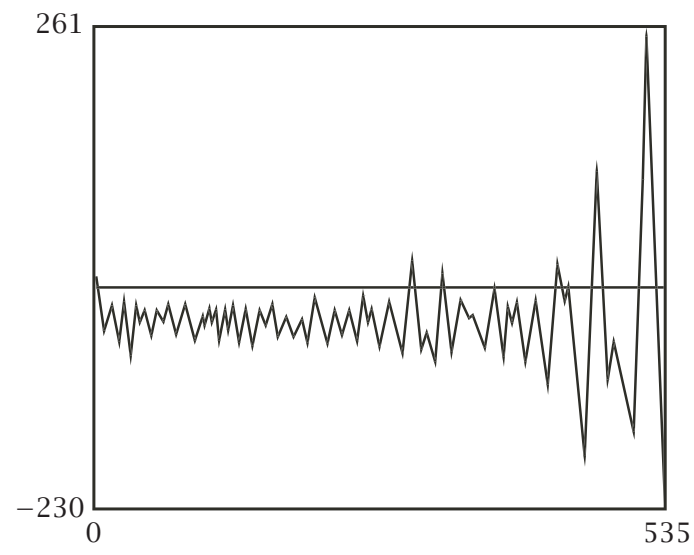

FiguRE 5.1. Plot of the original series $X_{2}(t)$.

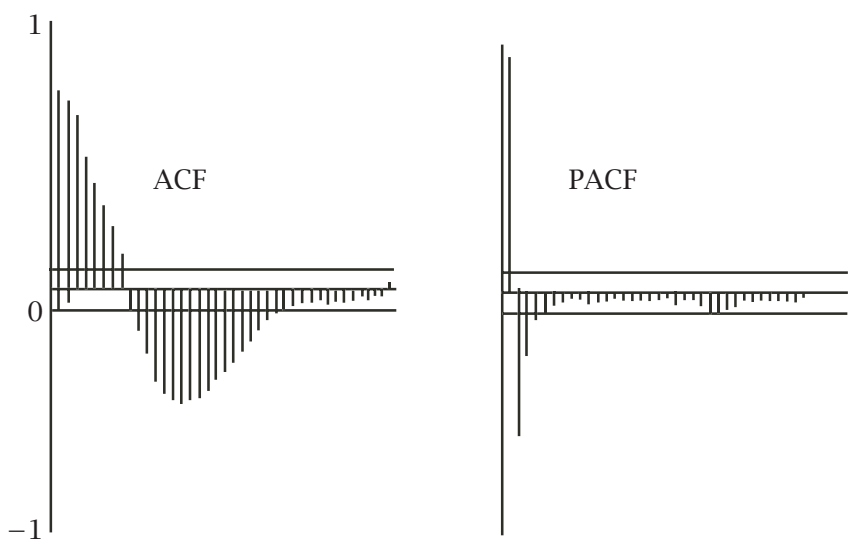

Figure 5.2. The ACF and PACF of $X_{2}(t)$.

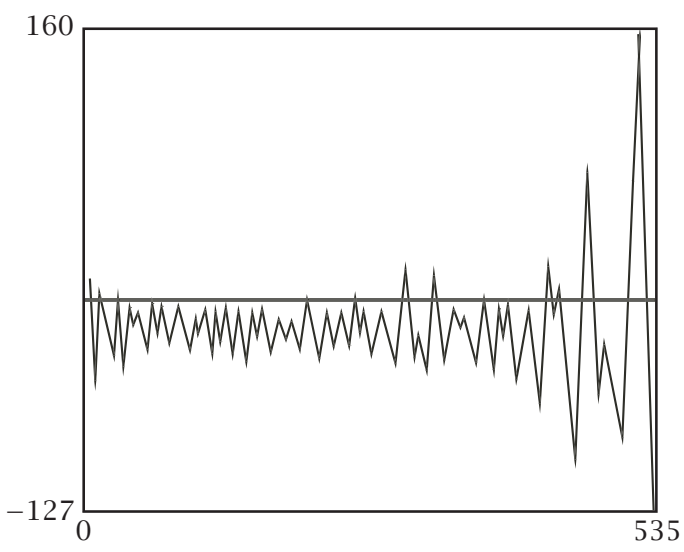

FiguRE 5.3. Plot of the estimated $\hat{X}_{2}(t)$. 
then

$$
\begin{gathered}
X_{2}(t)=\varphi_{1}^{*} X_{1}(t-1)+\varphi_{2}^{*} X_{2}(t-2)+\varphi_{3}^{*} X_{3}(t-3)+w(t), \\
\operatorname{Var}(w(t))=H_{w}(t)=\sigma_{\varepsilon}^{2} \exp (2 \alpha t)=\beta \exp (2 \alpha t), \\
\operatorname{Var}\left(Y_{2}(t)\right)=\alpha_{0}=f\left(\varphi_{1}, \varphi_{2}, \varphi_{3}\right) \sigma_{\varepsilon}^{2}, \quad \text { a constant, }
\end{gathered}
$$

where

$$
\begin{gathered}
f\left(\varphi_{1}, \varphi_{2}, \varphi_{3}\right)=\frac{\sigma_{\varepsilon}^{2}\left[\varphi_{3}\left(\varphi_{1}+\varphi_{3}\right)-\left(1-\varphi_{2}\right)\right]}{K}, \\
K=\left\{\varphi_{1}^{2}+\varphi_{2}^{2} \varphi_{3}^{2}+2 \varphi_{1} \varphi_{2} \varphi_{3}+\left(\varphi_{2}^{2}+\varphi_{3}^{2}+\varphi_{1} \varphi_{2} \varphi_{3}-1\right)\left(1-\varphi_{2}\right)\right. \\
\left.+\left(\varphi_{1} \varphi_{2}+\varphi_{3}+\varphi_{1}^{2} \varphi_{3}-\varphi_{3}^{3}\right)\left(\varphi_{1}+\varphi_{3}\right)\right\} .
\end{gathered}
$$

For any fixed value of $t$,

$$
\operatorname{Var}(X(t))=\exp (2 \alpha t) \operatorname{Var}(Y(t)) .
$$

For the given values of series $X_{2}(t)$ (available from the authors on request), the estimates $\hat{\varphi}_{1}^{*}, \hat{\varphi}_{2}^{*}, \hat{\varphi}_{3}^{*}, \hat{\beta}=\hat{\sigma}_{\varepsilon}^{2}$, and $\hat{\alpha}$ were obtained using the maximum likelihood (ML) method (see the appendix for details). The estimates are

$$
\begin{gathered}
\hat{\varphi}_{1}^{*}=230580, \quad \hat{\varphi}_{2}^{*}=-1.78829, \quad \hat{\varphi}_{3}^{*}=-0.45799, \\
\hat{\beta}=\hat{\sigma}_{\varepsilon}^{2}=321187, \quad \hat{\alpha}=0.0034 .
\end{gathered}
$$

Thus the estimated model for $X_{2}(t)$ series is

$$
\begin{gathered}
\hat{X}_{2}(t)=2306 X_{2}(t-1)-1.788 X_{2}(t-1)-0.458 X_{3}(t-1), \\
\operatorname{Var}\left(\hat{X}_{2}(t)=\exp (2 \hat{\alpha} t) \operatorname{Var}(Y(t))\right), \quad \text { at fixed } t, \\
\operatorname{Var}(\hat{Y}(t))=\hat{\beta} f\left(\hat{\varphi}_{1}, \hat{\varphi}_{2}, \hat{\varphi}_{3}\right),
\end{gathered}
$$

where

$$
\begin{gathered}
\hat{\varphi}_{1}=\hat{\varphi}_{1}^{*} e^{-\hat{\alpha}}, \quad \hat{\varphi}_{2}=\hat{\varphi}_{2}^{*} e^{-2 \hat{\alpha}}, \quad \hat{\varphi}_{3}=\hat{\varphi}_{3}^{*} e^{-3 \hat{\alpha}}, \\
\operatorname{Var}(\hat{w}(t))=\hat{\beta} \exp (2 \hat{\alpha} t), \quad \text { at fixed } t
\end{gathered}
$$

The estimated series $\hat{X}_{2}(t)$, its ACF, and PACF are plotted in Figures 5.3, 5.4a, and $5.4 \mathrm{~b}$, respectively.

INFERENCE 5.1. On comparing the plots of the original series $\hat{X}_{2}(t)$, its ACF, and PACF in Figures 5.1 and 5.2 with the corresponding plots of the estimated series $\hat{X}_{2}(t)$, its ACF and PACF in Figures 5.3, 5.4a, and 5.4b, one may note the striking similarity.

Thus based on (i) and (ii) above we may infer that the modelling of series $X_{2}(t)$, following the procedures outlined in Sections 3 and 4, is more than satisfactory.

In the following subsection, we consider the modelling series $X_{3}(t)$.

5.2. Modelling of series $X_{3}(t)$. The series of $X_{3}(t)$ consisting of 1051 observations is plotted in Figure 5.5. Its ACF and PACF are plotted in Figures 5.6a and 5.6b, respectively. 


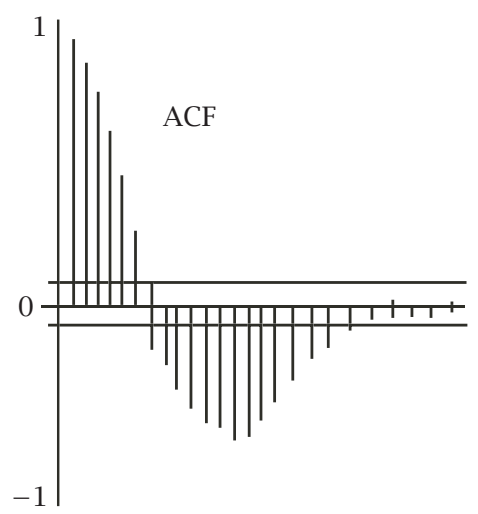

(a) Plot of the ACF of $\hat{X}_{2}(t)$.

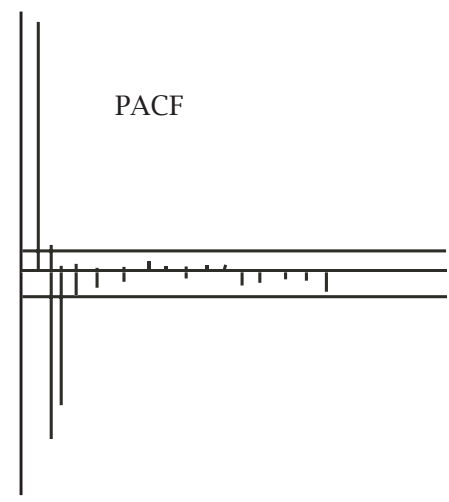

(b) Plot of the PACF of $\hat{X}_{2}(t)$.

FIGURE 5.4

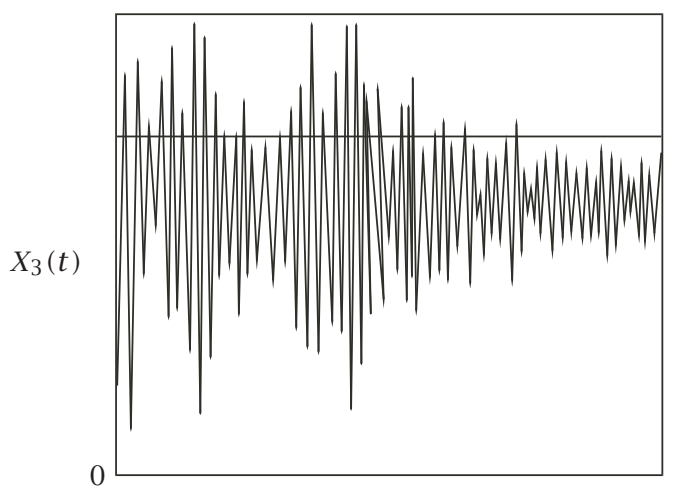

FIGURE 5.5 The plot of series $X_{3}(t)$.

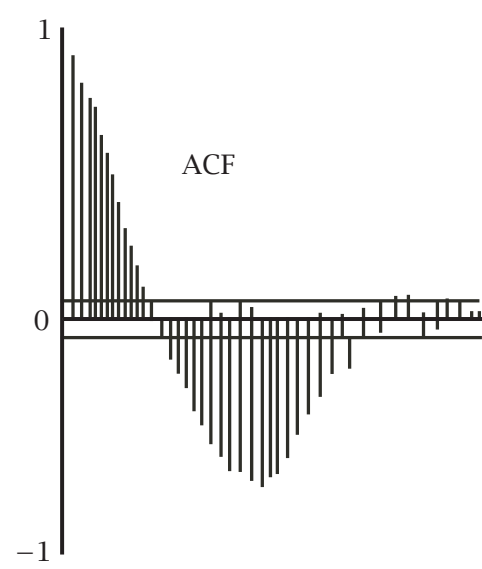

(a) The ACF of series $X_{3}(t)$.

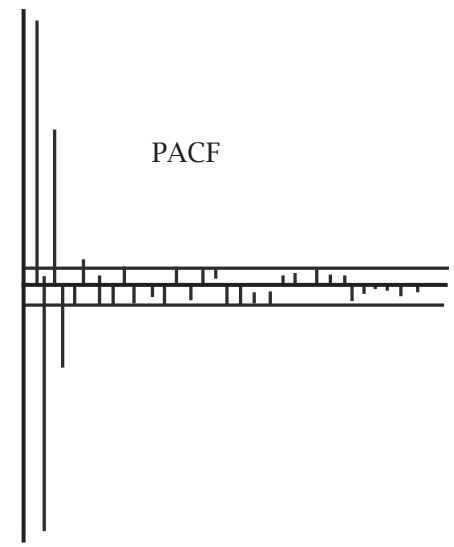

(b) The PACF of series $X_{3}(t)$.

FIGURE 5.6 
From Figures 5.5 and 5.6, it appears that the series in question represents a pure $\mathrm{AR}(4)$ process. Consequently, using ITSM, we fitted three models to $X_{3}(t)$, namely, $\mathrm{AR}(3), \mathrm{AR}(4)$, and $\mathrm{AR}(5)$ and obtained the respective AICC-values along with the corresponding maximum likelihood estimates. The AICC-values are shown in Table 5.1.

Through the AICC-value corresponding to AR(5) model is the minimal, however, in view of the parsimony and the PACF, we chose AR(4), namely,

$$
X_{3}(t)=2.670 \times(t-1)-2.81 \times(t-2)+1.418 \times(t-3)-0.328 \times(t-4)+\varepsilon(t) .
$$

The series $X_{1}(t)$ can be analyzed similarly.

TABLE 5.1

\begin{tabular}{ll}
\hline Model & AICC-value \\
\hline $\mathrm{AR}(3)$ & $0.188909 E+05$ \\
$\mathrm{AR}(4)$ & $0.177846 E+05$ \\
$\mathrm{AR}(5)$ & $0.177086 E+05$ \\
\hline
\end{tabular}

\section{Appendix}

Estimation of parameters. Consider a pure zero-mean AR(3) model defined by

$$
X(t)=\varphi_{1} X(t-1)+\varphi_{2} X(t-1)+\varphi_{3} X(t-3)+w(t),
$$

where $E X(t)=0, E X^{2}(t)<\infty$, and $E(w(t) w(s))=\delta_{t s} \sigma_{w}^{2}(t), \delta_{t s}$ is the Kronecker delta. Furthermore, it is assumed that $E\left(X_{t} w(s)\right)=0$ for $s>t$ and the process $X(t)$ is nonstationary in variance, that is, $\sigma_{w}^{2}(t)$ is a function of time; however, it is assumed to be bounded and bounded away from zero.

Defining $\underset{\sim}{X}(1)=(X(t), X(t-1), X(t-2))^{T}, \underset{\sim}{w}(t)=(w(t), 0,0)^{T}$, and $\Phi=\left[\begin{array}{ccc}\varphi_{1} & \varphi_{2} & \varphi_{3} \\ 1 & 0 & 0 \\ 0 & 1 & 0\end{array}\right]$, (3.2) can be expressed as a 3-dimensional nonstationary-in-variance vector AR(1) process, that is,

$$
\underset{\sim}{X}(t)-\underset{\sim}{X}(t-1)=\underset{\sim}{w}(t)
$$

Given $\underset{\sim}{X}(1), \underset{\sim}{X}(2), \ldots, \underset{\sim}{X}(n)$, it can be shown easily (see Tyssedal and Tjostheim [5]) that the weighted least squares estimate of $\Phi$ is given by

$$
\hat{\Phi}=n^{-1} \sum_{t=1}^{n} \frac{\underset{\sim}{X}(t) \underset{\sim}{X^{T}}(t-1)}{H_{w}(t)}\left[n^{-1} \sum_{t=1}^{n} \frac{\underset{\sim}{X}(t-1) \underset{\sim}{X^{T}}(t-1)}{H_{w}(t)}\right]^{-1},
$$

where $H_{w}(t)=\sigma_{w}^{2}(t)$. If the $W(t)$ are assumed normally distributed, then $\hat{\Phi}$ is asymptotically equivalent to the maximum likelihood estimate of $\Phi$. The nonweighed least square estimate of $\Phi$ is given by

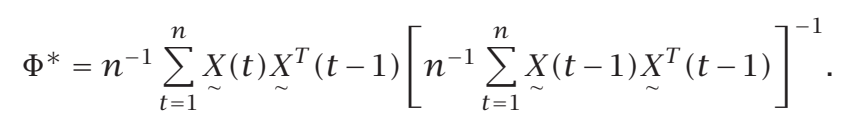


Given $w(1), w(2), \ldots, w(n)$, the likelihood function is

$$
L=(2 \pi)^{-n / 2} \prod_{t=1}^{n}\left(H_{w}(t)\right)^{-1 / 2} \exp \left[-\frac{1}{2} \sum_{t=1}^{n} w^{2}(t)\left(H_{w}(t)\right)^{-1}\right] .
$$

Assuming $H_{w}(t)=\beta \exp (\alpha t), 0<\alpha<1, B>0$, the log likelihood is

$$
\ln \alpha=K-\frac{1}{2} \sum_{t=1}^{n} \ln \beta-\frac{1}{2} \alpha \sum_{t=1}^{n} t-\frac{1}{2} \sum_{t=1}^{n} w^{2}(t) \beta^{-1} e^{-\alpha t} .
$$

Replacing $w(t)$ by $\left(X(t)-\varphi_{1} X(t-1)-\varphi_{2} X(t-2)-\varphi_{3} X(t-3)\right)$ in (A.6) and then, differentiating with respect to $\alpha$ and $\beta$ we obtain

$$
\begin{gathered}
\sum_{t=1}^{n} t=\hat{\beta}^{-1} \sum_{t=1}^{n}\left(X(t)-\varphi_{1}^{*} X(t-1)-\varphi_{2}^{*} X(t-2)-\varphi_{3}^{*} X(t-3)\right)^{2} e^{-\hat{\alpha} t}, \\
\hat{\beta}=n^{-1} \sum_{t=1}^{n}\left(X(t)-\varphi_{1}^{*} X(t-1)-\varphi_{2}^{*} X(t-2)-\varphi_{3}^{*} X(t-3)\right)^{2} e^{-\hat{\alpha} t},
\end{gathered}
$$

respectively, where $\varphi_{1}^{*}, \varphi_{2}^{*}$, and $\varphi_{3}^{*}$ are the preliminary estimates of $\varphi_{1}, \varphi_{2}$, and $\varphi_{3}$ obtained from (A.4) for known $X(t), X(t-1)$, and $X(t-2)$. For a convergent solution we suggest the following steps:

(1) choose an initial value of $\alpha$ between 0 and 1 , say, $\hat{\alpha}_{0}$. Using $\hat{\alpha}_{0}, \hat{\beta}_{0}$ can be estimated from (A.8);

(2) using $\hat{\beta}_{0}, \alpha$ can be re-estimated from (A.7) and then $\beta$ is re-estimated from (A.8) using a new estimate $\hat{\alpha}$;

(3) steps 1 and 2 are repeated until converging estimates of $\alpha$ and $\beta$ for the preliminary estimates $\Phi^{*}$;

(4) $\Phi^{*}$ is re-estimated from (A.3) using these convergent estimates of $\alpha$ and $\beta$;

(5) steps 1 to 4 are repeated until the convergent estimates of $\Phi^{*}, \alpha$, and $\beta$ are obtained.

AcKnowledgments. The authors are thankful to Dr Greg Houseman of the Department of Earth Sciences, Monash University, Clayton Vic. 3168, Australia, for kindly supplying the seismographs and the data of the earthquake which occurred at Kobe, Japan on 17 January 1995. The authors wish to thank Prof E. C. Reynardt and Prof F. E. Steffens for their support to make this project successful.

\section{REFERENCES}

[1] G. E. P. Box and G. M. Jenkins, Time Series Analysis: Forecasting and Control, Holden-Day, California, 1976.

[2] G. R. Dargahi-Noubary, P. J. Laycock, and T. Subba Rao, Non-linear stochastic models for seismic events with applications in event identification, Geophysical Journal of Royal Astronomical Society 55 (1978), 655-668.

[3] D. Tjostheim, Autoregressive representation of seismic $P$-wave signals with an application to the problem of short-period discriminates, Geophysical Journal of Royal Astronomical Society 43 (1975), 269-291.

[4] , Some autoregressive models for short-period seismic noise, Bull. Seismol. Soc. Amer. 65 (1975), 677-691. 
[5] J. S. Tyssedal and D. Tjostheim, Autoregressive processes with a time dependent variance, J. Time Ser. Anal. 3 (1982), no. 3, 209-217.

N. Singh: Department of MATHematics, Monash University, VIC 3168, Australia Current address: DePARTMENT OF STATISTICS, 0003 UNISA, SOUTH AFRICA

D. TAmpubolon: Department of Mathematics, Monash University, VIC 3168, AuSTRALIA

V. S. S. YADAVAlli: DePartment OF STATiSTics, 0003 UNISA, SOUTH AFRICA E-mail address: yadavvss@unisa.ac.za 


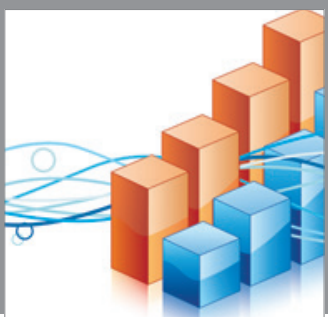

Advances in

Operations Research

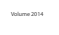

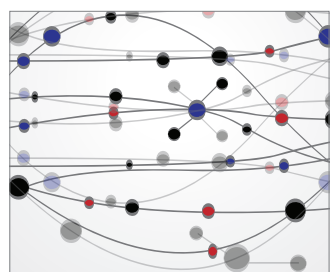

\section{The Scientific} World Journal
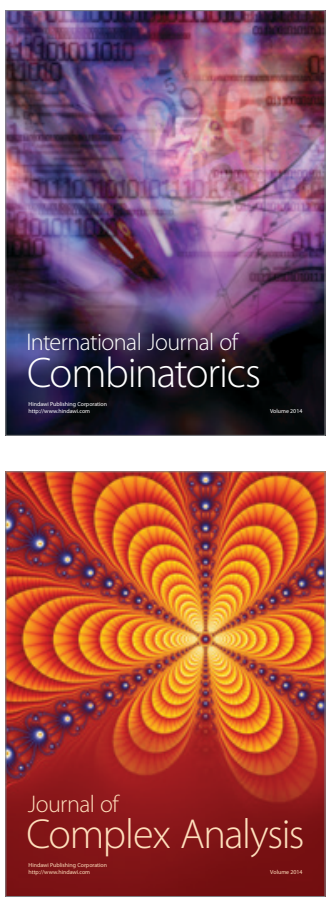

International Journal of

Mathematics and

Mathematical

Sciences
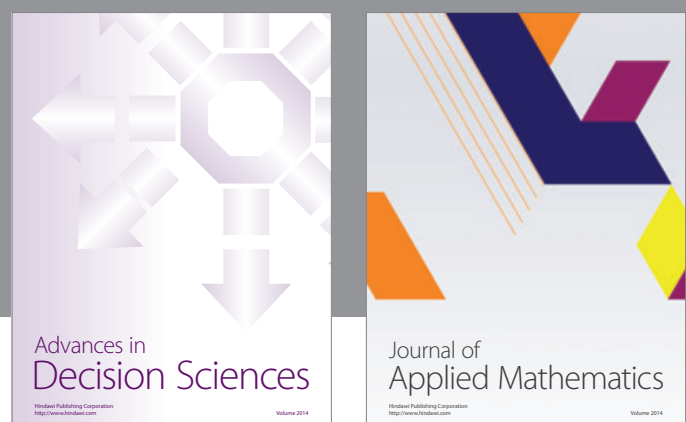

Journal of

Applied Mathematics
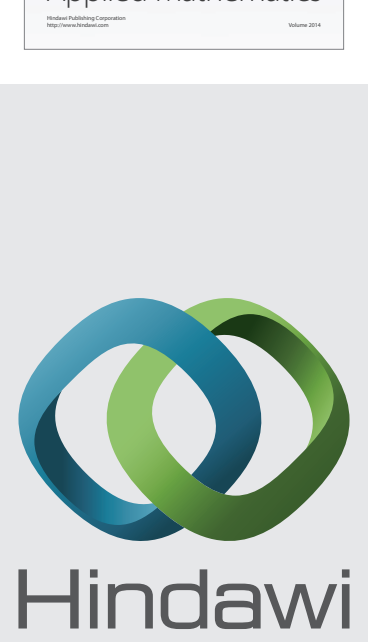

Submit your manuscripts at http://www.hindawi.com
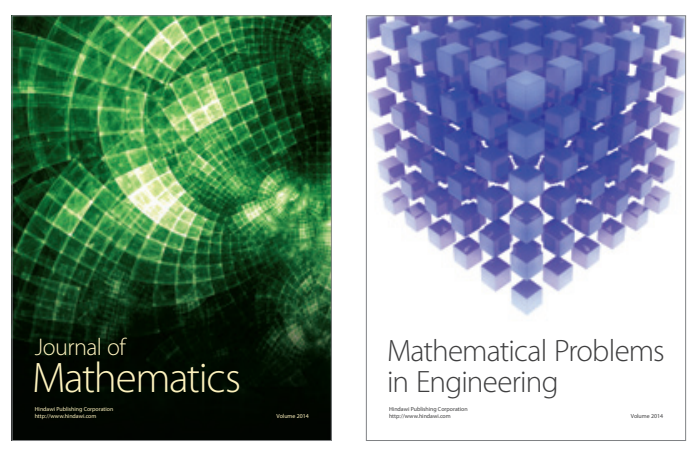

Mathematical Problems in Engineering
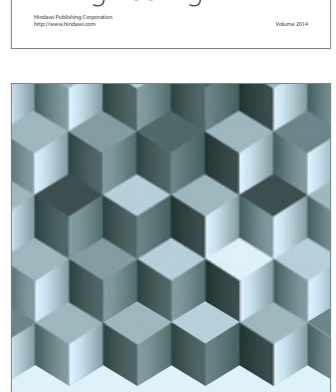

Journal of

Function Spaces
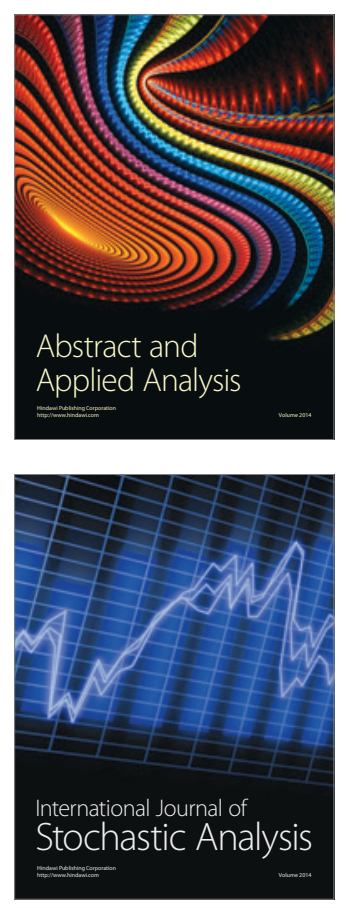

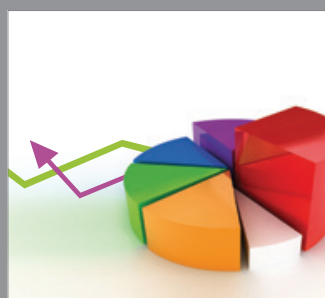

ournal of

Probability and Statistics

Promensencen
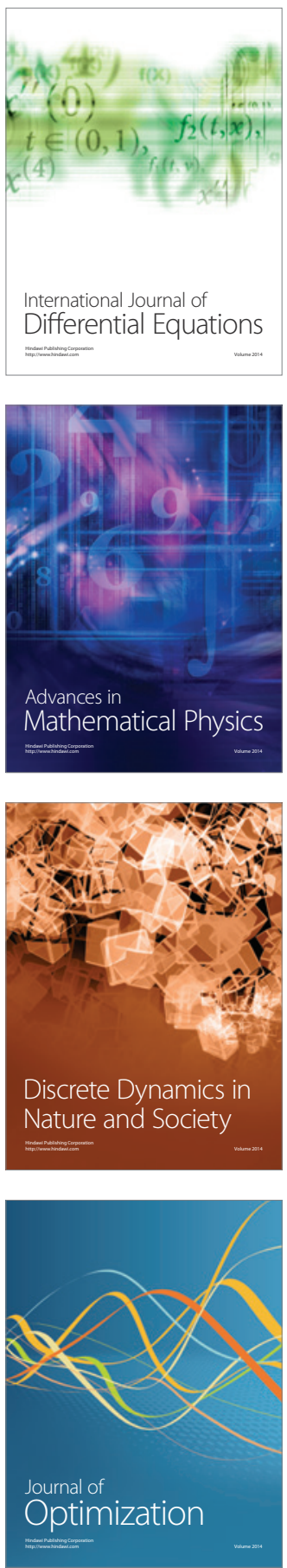